\title{
Iron Supplementation in Non-Anemic Pregnancy and Risk of Developing Gestational Diabetes Mellitus
}

\author{
Preeyaporn Jirakittidul ${ }^{\mathrm{a}}$ e, Supatra Sirichotiyakul ${ }^{\mathrm{b}}$, Chidchanok Ruengorn ${ }^{\mathrm{c}}$, Siwaporn Siripenpong ${ }^{\mathrm{d}}$, \\ Arinporn Imruetaicharoenchok ${ }^{\mathrm{d}}$, Busaba Wiriyasirivaj ${ }^{\mathrm{d}}$
}

\begin{abstract}
Background: Routine iron supplementation for all pregnant women in order to reduce adverse neonatal outcomes has been a standard practice in developing countries, including Thailand. However, there is evidence that excess iron can affect glucose metabolism and may increase risk of gestational diabetes mellitus (GDM). This study aims to investigate the association of iron supplementation starting from early gestation and the risk of GDM in non-anemic women.
\end{abstract}

Methods: This retrospective cohort study included non-anemic pregnant Thai women who received their first antenatal care and delivered at Vajira Hospital (Bangkok, Thailand) during January 2008 to December 2009. All pregnant women underwent oral glucose tolerance test during gestational weeks $24-28$. The proportions of ongoing GDM and birth outcomes were compared between the early oral iron supplementation group (before 16 completed weeks) and the control group (after 16 weeks).

Results: There were 1,935 non-anemic pregnant women, 966 in the early supplement group and 969 in the control group. The early supplement group had significantly higher prevalence of GDM than the control group did $(9.7 \%$ vs. $5.6 \%$, RR: $1.83 ; 95 \%$ CI: $1.29-2.59)$. No significant differences in maternal anemia, gestational age at birth, or neonatal birth weight were observed between groups.

Conclusions: Early antenatal iron supplementation in non-anemic pregnant women was found to be associated with significantly increased risk of GDM.

Manuscript submitted December 13, 2018, accepted December 27, 2018

aDepartment of Obstetrics and Gynecology, Faculty of Medicine Siriraj Hospital, Mahidol University, Bangkok, Thailand

${ }^{\mathrm{b}}$ Department of Obstetrics and Gynecology, Faculty of Medicine, Chiang Mai University, Chiang Mai, Thailand

'Department of Pharmaceutical Care, Faculty of Pharmacy, Chiang Mai University, Chiang Mai, Thailand

${ }^{\mathrm{d} D e p a r t m e n t ~ o f ~ O b s t e t r i c s ~ a n d ~ G y n e c o l o g y, ~ F a c u l t y ~ o f ~ M e d i c i n e ~ V a j i r a ~ H o s p i-~}$ tal, Navamindradhiraj University, Bangkok, Thailand

${ }^{\mathrm{e}}$ Corresponding Author: Preeyaporn Jirakittidul, Department of Obstetrics and Gynaecology, Faculty of Medicine Siriraj Hospital, Mahidol University, 2 Wanglang Road, Bangkoknoi, Bangkok 10700, Thailand.

Email: preeyajira@hotmail.com

doi: https://doi.org/10.14740/jem543
Keywords: Iron supplementation; Non-anemic pregnancy; Gestational diabetes mellitus; Thai

\section{Introduction}

Iron-deficiency anemia is the most common nutritional problem during pregnancy, particularly in developing countries [1]. Maternal anemia is strongly associated with many adverse pregnancy outcomes [1]. Prophylactic iron supplementation is routinely recommended to all pregnant women in order to reduce maternal anemia, preterm delivery, low birth weight infants and perinatal mortality rate $[1,2]$. Although there are many benefits from iron supplementation during pregnancy, there is a concern that women without anemia may be exposed to potential risk of unnecessary iron loading from routine prescription. It has been notified that such routine practice may build up the mother's iron stores, increase the risk of hemoconcentration by the end of gestation, and increase blood viscosity to the level that impairs utero-placental blood flow. In addition, this excess iron may increase iron free radical and the associated oxidative stress [3]. This is further complicated by the increasing evidences about the association between iron status and adverse pregnancy outcomes such as low birth weight infants, preterm delivery, preeclampsia and gestational diabetes mellitus (GDM) [2-4].

The existing evidence demonstrates that pancreatic betacell is susceptible to iron-related oxidative free radicals, which contribute to GDM [3]. In addition, some studies had shown that pregnant women with higher hemoglobin or higher ferritin level might have a higher risk for preeclampsia and GDM [5-7]. Indeed, the incidence of GDM in women with iron deficiency anemia was not only lower than that in the non-anemic women, but it was also significantly lower than that in women with thalassemia trait [8]. However, impact of iron supplementation on the risk of GDM is still inconclusive. Some studies found the association of iron supplementation with glucose impairment and hypertensive disorder in pregnancy $[3,9,10]$. On the other hand, some studies demonstrated that iron supplement starting from early pregnancy did not increase the risk of GDM; instead, it had benefited in other pregnancy outcomes $[6,11,12]$.

Because GDM is the most common medical complication of pregnancy that causes poor maternal and fetal outcomes [13], 
and that the harmful effects of iron supplementation in nonanemic pregnant women are not fully understood highlights the need to investigate the relationship between early antenatal iron supplementation and the risk of developing GDM. Accordingly, the aim of the present study was to investigate the association between early antenatal iron supplementation and the risk of developing GDM in non-anemic pregnant Thai women.

\section{Materials and Methods}

The protocol for this retrospective cohort study was approved by the Bangkok Metropolitan Administration Ethics Committee for Research Involving Human Subjects. The requirement to obtain written informed consent was waived given the retrospective and anonymous nature of this study. The present study included non-anemic pregnant Thai women who received their first antenatal care and delivered their babies at the Faculty of Medicine Vajira Hospital, Navamindradhiraj University, Bangkok, Thailand during the January 2008 to December 2009 study period, when all pregnant women underwent GDM screening by using 50-g 1-h glucose challenge test (GCT) during 24 - 28 weeks of gestation. Individuals who had positive GCT (blood glucose level $\geq 140 \mathrm{mg} / \mathrm{dL}$ ) were scheduled for the diagnostic 100-g oral glucose tolerance test (OGTT). The GDM diagnosis was based on The National Diabetes Data Group criteria [13].

We included all singleton pregnant women who had their first antenatal care at Vajira Hospital and were non-anemic (hemoglobin levels $\geq 11 \mathrm{~g} / \mathrm{dL}$ from the first antenatal blood test result). We excluded those who were thalassemia trait, had known medical complicating pregnancy (such as overt diabetes mellitus, chronic hypertension, thyroid disease), and had positive GCT but did not undergo an OGTT. The study population was divided into two groups according to the gestational age when iron supplementation was started, i.e. the early supplement and the control groups. The early supplement group was women who started taking oral iron supplementation (200 $\mathrm{mg}$ of ferrous fumarate) prior to 16 completed weeks of gestation while the control group was those who started later. The proportion of gestational diabetes mellitus was compared between the two groups.

Collected data included maternal demographic data, clinical characteristics, and risk factors for gestational diabetes, such as advanced maternal age, pre-pregnancy obesity, family history of diabetes in first-degree relatives, history of fetal macrosomia, and unexplained fetal demise. Iron supplementation information was obtained from the obstetric clinical record and the hospital computer-based recording system. Drug adherence was calculated using the following formula: Iron supplementation adherence $\%=$ (total number of prescribed iron tablets during the antenatal care period/total days during the antenatal period that iron was prescribed) $\times 100$.

The adherence of $\geq 75 \%$ was considered good adherence.

Maternal delivery details, hemoglobin levels, and neonatal outcomes were recorded according to standard protocol in the labor room. Anemia at delivery was defined as maternal hemoglobin level of lower than $11 \mathrm{~g} / \mathrm{dL}$.

Sample size was calculated based on the incidence of
GDM in previous studies which showed the incidence of $7.84 \%$ in the early supplement group and $4.16 \%$ in the control group $[9,14]$. Using a formula to compare proportion between two groups with a power of $90 \%$ and a 0.05 level of significance, a sample size of 875 per group was required.

Statistical analysis was performed using Stata statistical software (version 14) (StataCorp LLC, College Station, TX, USA). Demographic data and glucose values are shown as mean \pm standard deviation (SD) for continuous variables and as number (n) with percentage (\%) for categorical variables. Binary regression analysis and multinomial regression analysis were used to estimate relative risks (RR) of having gestational diabetes in the early supplement group compared to the control group. A $\mathrm{P}$-value less than 0.05 was considered significant for all tests.

\section{Results}

A total of 1,935 singleton pregnant women were recruited in this study, 966 in the early iron supplement group and 969 in the control group. The baseline characteristics of the two groups were shown in Table 1. There were no significant difference between the two groups in terms of hemoglobin level at booking, gravidity, previous GDM, previous fetal macrosomia $(\geq 4,000$ g) and history of unexplained fetal demise. Mean maternal age and a proportion of maternal age of older than 30 years were significantly higher in the early supplement group. There were also significant differences in body mass index (BMI), pre-pregnancy overweight, family history of diabetes in first-degree relative between the two groups. The proportion of good drug adherence was not significant difference between the groups (early supplement group $=71.4 \%$; control group $=68.9 \% ; \mathrm{P}=0.253$ ).

Tables 2 and 3 show the GDM outcome. The overall prevalence of GDM was $7.6 \%$ (148 in 1,935 women), which was significantly higher in the early supplement group than in the control group (9.7\% vs. 5.6\%, RR: 1.83, 95\% CI: 1.29 - 2.59; $\mathrm{P}<0.001)$. The risk increased only in the sub-type GDM-A1, which does not require anti-hyperglycemic agents (RR: 2.25, 95\% CI: 1.49 - 3.39; P $<0.001$ ), but not in the sub-type GDM$\mathrm{A} 2$, which requires medical treatment (RR: $1.05,95 \% \mathrm{CI}: 0.55$ - 2.00; $\mathrm{P}=0.883$ ). After adjustment for confounding factors present in the background characteristics or in the theoretical concept, the early supplement group still had a significant higher risk of GDM, especially for the sub-type GDM-A1, as shown in Table 3.

Table 4 demonstrates obstetric outcomes. Infant birth weight, maternal hemoglobin level and gestational age at birth were not significant differences between the two groups. The risk of maternal anemia at delivery was not significantly affected by iron supplementation protocol. None of the infants had an Apgar score at 10 min of less than 7.

\section{Discussion}

The prevalence of GDM using universal screening in the present study was nearly $8 \%$, the prevalence of which was consistent with an overall prevalence of GDM that ranges from 
Table 1. Background Characteristics Compared Between the Early and Late Iron Supplementation Groups

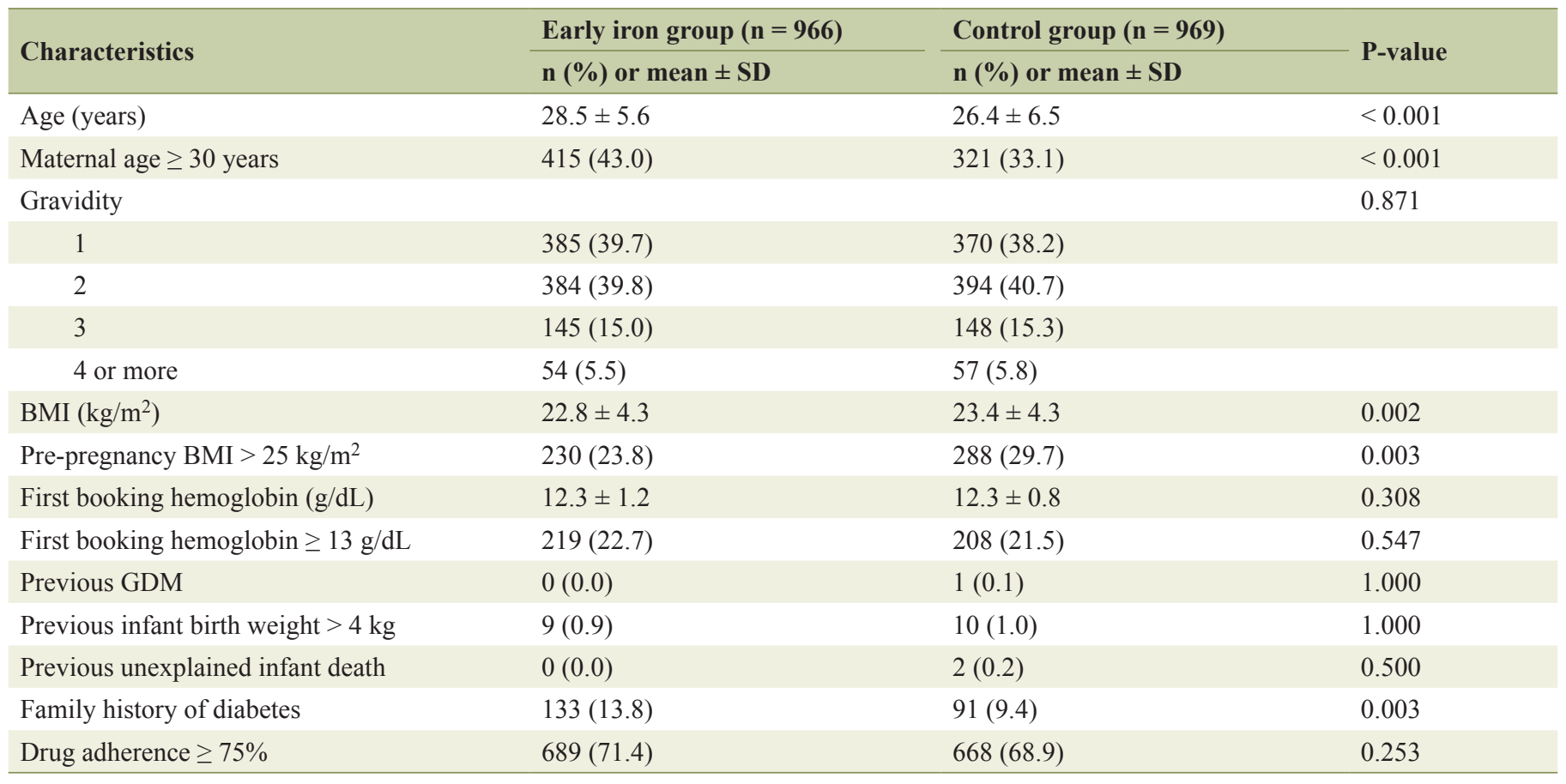

A P-value < 0.05 indicates statistical significance. BMI: body mass index; GDM: gestational diabetes mellitus; SD: standard deviation.

Table 2. The Incidence of Gestational Diabetes Mellitus Compared Between the Early and Late Iron Supplementation Groups

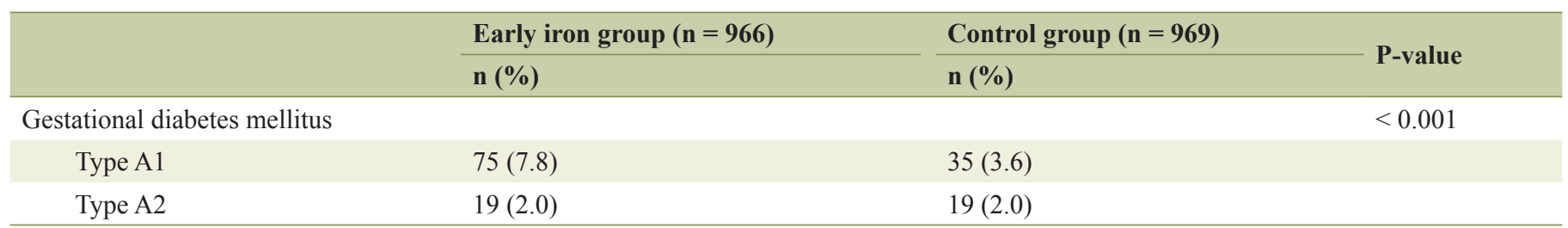

A P-value < 0.05 indicates statistical significance. A1: subtype of gestational diabetes mellitus which does not require anti-hyperglycemic drug; A2: subtype of gestational diabetes mellitus which requires anti-hyperglycemic drug.

Table 3. Univariable and Multivariable Analysis of Association Between Early Antenatal Iron Supplementation and GDM

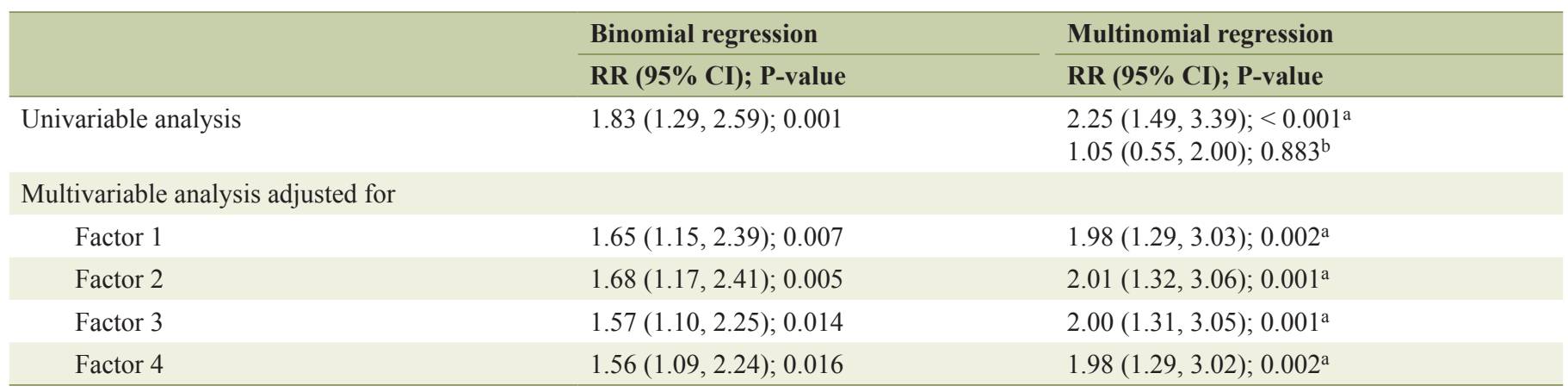

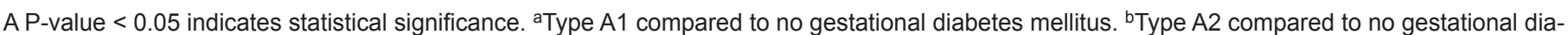
betes mellitus. Factor 1: maternal age, pre-pregnancy BMI, history of DM in first-degree relatives; Factor 2: maternal age $\geq 30$ years, pre-pregnancy $\mathrm{BMI} \geq 25 \mathrm{~kg} / \mathrm{m}^{2}$, history of DM in first-degree relatives; Factor 3: maternal age $\geq 30$ years, pre-pregnancy BMI $\geq 25 \mathrm{~kg} / \mathrm{m}^{2}$, history of DM in first-degree relatives, previous GDM, previous infant birth weight $>4 \mathrm{~kg}$; Factor 4 : maternal age $\geq 30$ years, pre-pregnancy BMI $\geq 25 \mathrm{~kg} / \mathrm{m}^{2}$, history of DM in first-degree relatives, previous GDM, previous infant birth weight $>4 \mathrm{~kg}$, drug adherence, first hemoglobin level result. BMl: body mass index; Cl: confidence interval; DM: diabetes mellitus; GDM: gestational diabetes mellitus; RR: relative risk. 
Table 4. Birth Outcomes Compared Between the Early and Late Iron Supplementation Groups

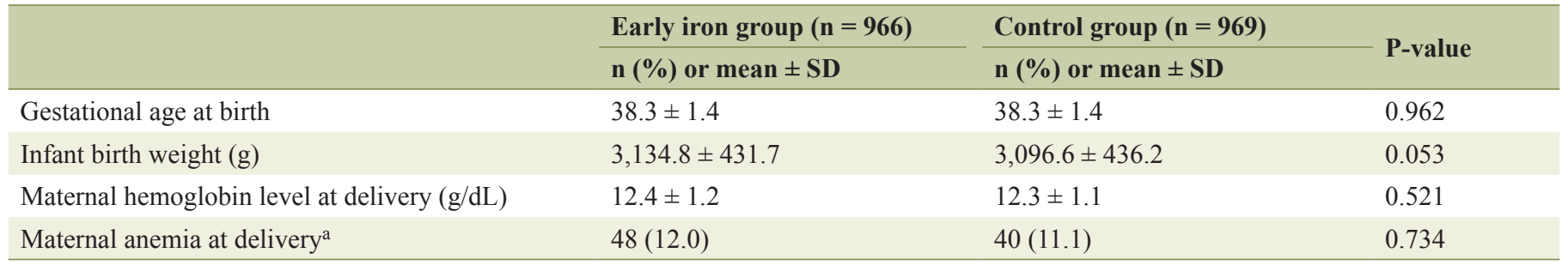

A P-value < 0.05 indicates statistical significance. ${ }^{a}$ Hemoglobin level $<11 \mathrm{~g} / \mathrm{dL}$. SD: standard deviation.

$1 \%$ to $25 \%$ worldwide depending on the ethnicity of mother and diagnostic criteria [13].

In the present cohort study, we directly investigated the possible association between iron supplement starting early (before 16 completed weeks of gestation) and the risk of GDM in non-anemic pregnant Thai women. We discovered that early iron supplementation prior to 16 completed weeks of gestation increased the risk of GDM by 1.5 to 2 folds in women who had good hemoglobin level at the beginning of pregnancy. Even after the adjustment for several reasonable confounding factors, such impact was still existed. Our result was consistent with several previous epidemiological studies that have reported an association between body iron status and GDM [6, 7, 15, 16]. They found that high maternal hemoglobin level and/or high serum ferritin levels were the risk factor for GDM [15]. On the other hand, some studies were conducted in order to investigate whether or not iron supplementation in non-anemic pregnancy may increase the risk of development of GDM. A case control study including 500 women with GDM and 500 women with normoglycemia reported higher odds of GDM about 2 to 3 folds in the iron supplement women compared with the non-supplement ones; the odds were independent to other confounders such as maternal age, diabetes in first-degree relatives, pre-pregnancy BMI, and duration of iron supplementation [17]. A prospective cohort study in pregnant women at increased risk for GDM reported an increased risk of GDM in women who received a high iron intake during pregnancy, especially in non-anemic ones [18]. In contrast, some randomized controlled studies concluded that early iron supplementation starting at gestational age less than 16 weeks did not increase risk for GDM $[11,12]$. However, the participants in these studies included both anemic and non-anemic pregnant women who might respond differently to iron consumption.

There were some studies showing that pregnant women without antenatal iron supplement had adverse obstetric outcomes including maternal anemia, preterm delivery and low birth weight infant $[11,16,19]$. However, in the present study, the early (before 16 weeks gestation) and the later (after 16 weeks gestation) iron supplementation had comparable infants' birth weight, length of gestational period, and incidence of maternal anemia at delivery. The aforementioned evidences suggested that iron supplementation had beneficial effects on obstetric outcomes and the supplementation in non-anemic women could be started later, i.e. after 16 completed weeks of gestation, as such protocol did not increase GDM risk and did not attenuate the benefit on obstetric outcomes.

Limitation of the present study was the study design. With its retrospective nature, the iron status of the study population was not available. Non-anemic women could have normal, replete, or deficient iron storage; each status would differently affect the response of iron supplementation. In addition, the data of dietary iron intake and micronutrient co-supplementation during gestation were not available either. These factors would have modified the effects of iron supplementation. Even though, our study had several strengths. It was the first large cohort study that had adequate power to demonstrate the harm of iron supplement in non-anemic pregnant women as such practice might induce GDM. The robustness of the result was confirmed as the risk of GDM still existed after multiple regression analysis adjusting for several reasonable confounding factors. Moreover, the universal screening for GDM in our antenatal clinic provided the reliable diagnosis of the primary outcome, i.e. GDM.

\section{Conclusions}

In summary, it seems that iron intake during early gestation might be a factor which increases the risk of GDM especially in women with sufficient iron storage or non-anemia. Our study suggests that routine iron supplementation should be reconsidered, especially in non-anemic women. To confirm the hypothesis, there is a need for a large prospective study (ideally a randomized controlled trial) with reliable and comprehensive information on iron intake from food and drug prescriptions during pregnancy accompanied by serum measurements to determine the level of body iron stores.

\section{Acknowledgments}

The authors gratefully acknowledge Prof. Manee Rattanachaiyanont and Mr. Kevin P. Jones for assistance with proofreading.

\section{Conflict of Interest}

All authors declare no conflict of interest.

\section{Funding Support}

This was an unfunded study. 


\section{References}

1. Black RE, Victora CG, Walker SP, Bhutta ZA, Christian $\mathrm{P}$, de Onis M, Ezzati M, et al. Maternal and child undernutrition and overweight in low-income and middle-income countries. Lancet. 2013;382(9890):427-451.

2. Brannon PM, Taylor CL. Iron supplementation during pregnancy and infancy: uncertainties and implications for research and policy. Nutrients. 2017;9(12):1327.

3. Zhuang T, Han H, Yang Z. Iron, oxidative stress and gestational diabetes. Nutrients. 2014;6(9):3968-3980.

4. Friedrisch JR, Friedrisch BK. Prophylactic iron supplementation in pregnancy: a controversial issue. Biochem Insights. 2017;10:1178626417737738.

5. Phaloprakarn C, Tangjitgamol S. Impact of high maternal hemoglobin at first antenatal visit on pregnancy outcomes: a cohort study. J Perinat Med. 2008;36(2):115-119.

6. Fu S, Li F, Zhou J, Liu Z. The relationship between body iron status, iron intake and gestational diabetes: a systematic review and meta-analysis. Medicine (Baltimore). 2016;95(2):e2383.

7. Rawal S, Hinkle SN, Bao W, Zhu Y, Grewal J, Albert PS, Weir NL, et al. A longitudinal study of iron status during pregnancy and the risk of gestational diabetes: findings from a prospective, multiracial cohort. Diabetologia. 2017;60(2):249-257.

8. Lao TT, Ho LF. Impact of iron deficiency anemia on prevalence of gestational diabetes mellitus. Diabetes Care. 2004;27(3):650-656.

9. Ozyigit EA UM, Unlu S, Ozaksit G, Avsar F. The effect of oral iron supplementation on the glucose metabolism in non-anemic pregnant women: A prospective case-control study. UHOD. 2008;18:155-162.

10. Jirakittidul P, Sirichotiyakul S, Ruengorn C, Techatraisak $\mathrm{K}$, Wiriyasirivaj B. Effect of iron supplementation during early pregnancy on the development of gestational hypertension and pre-eclampsia. Arch Gynecol Obstet. 2018;298(3):545-550.

11. Chan KK, Chan BC, Lam KF, Tam S, Lao TT. Iron supplement in pregnancy and development of gestational diabetes - a randomised placebo-controlled trial. BJOG. 2009;116(6):789-797; discussion 797-788 .

12. Kinnunen TI, Luoto R, Helin A, Hemminki E. Supplemental iron intake and the risk of glucose intolerance in pregnancy: re-analysis of a randomised controlled trial in Finland. Matern Child Nutr. 2016;12(1):74-84.

13. Piper LK, Stewart Z, Murphy HR. Gestational diabetes. Obstetrics, Gynaecology \& Reproductive Medicine. 2017;27(6):171-176.

14. Yamasmit W, Chaithongwongwatthana S, Uerpairojkit B. A 50-g glucose challenge test: is there any diagnostic cutoff? J Med Assoc Thai. 2008;91(9):1309-1312.

15. Bowers KA, Olsen SF, Bao W, Halldorsson TI, Strom $M$, Zhang C. Plasma concentrations of ferritin in early pregnancy are associated with risk of gestational diabetes mellitus in women in the Danish national birth cohort. J Nutr. 2016;146(9):1756-1761.

16. Iqbal S, Ekmekcioglu C. Maternal and neonatal outcomes related to iron supplementation or iron status: a summary of meta-analyses. J Matern Fetal Neonatal Med. 2017:113.

17. Bo S, Menato G, Villois P, Gambino R, Cassader M, Cotrino I, Cavallo-Perin P. Iron supplementation and gestational diabetes in midpregnancy. Am J Obstet Gynecol. 2009;201(2):158 e151-156.

18. Helin A, Kinnunen TI, Raitanen J, Ahonen S, Virtanen SM, Luoto R. Iron intake, haemoglobin and risk of gestational diabetes: a prospective cohort study. BMJ Open. 2012;2(5):e001730.

19. Pena-Rosas JP, De-Regil LM, Garcia-Casal MN, Dowswell T. Daily oral iron supplementation during pregnancy. Cochrane Database Syst Rev. 2015;7:CD004736. 